\title{
Signal and noise in Gravity Recovery and Climate Experiment (GRACE) observed surface mass variations
}

Ernst J. O. Schrama, ${ }^{1}$ Bert Wouters, ${ }^{1}$ and David A. Lavallée ${ }^{2}$
Received 30 November 2006; revised 8 May 2007; accepted 18 May 2007; published 4 August 2007.

[1] The Gravity Recovery and Climate Experiment (GRACE) product used for this study consists of 43 monthly potential coefficient sets released by the GRACE science team which are used to generate surface mass thickness grids expressed as equivalent water heights (EQWHs). We optimized both the smoothing radius and the level of approximation by empirical orthogonal functions (EOFs) and found that $6.25^{\circ}$ and three modes are able to describe more than $73.5 \%$ of the variance. The EQWHs obtained by the EOF method describe all known variations in the continental hydrology, present-day ice sheet melting, and global isostatic adjustment. To assess the quality of the estimated grids, we constructed degree error spectra of EQWHs. We conclude that a significant part of the errors in GRACE can be explained by a scaling factor of 0.85 relative to degree error estimates provided by the GGM02C gravity model but that the present-day errors in the GRACE data are a factor 2 to 5 larger than forecasted by tide model differences and atmospheric pressure differences. Comparison to a network of 59 International GNSS Service (IGS) stations confined the filter parameter settings to three EOF modes and $5^{\circ}$ or $6.25^{\circ}$ smoothing radius. Residuals that remain after the EOF method do exhibit S2 aliasing errors and a semiannual continental hydrology signal contained in the Global Land Data Assimilation Systems (GLDAS) model. Further analysis of the residual EOF signal revealed alternating track correlation patterns that are partially explained by the GRACE covariance matrix and the handling of nuisance parameters in the GRACE data processing.

Citation: Schrama, E. J. O., B. Wouters, and D. A. Lavallée (2007), Signal and noise in Gravity Recovery and Climate Experiment (GRACE) observed surface mass variations, J. Geophys. Res., 112, B08407, doi:10.1029/2006JB004882.

\section{Introduction}

[2] The Gravity Recovery and Climate Experiment (GRACE) is a twin satellite configuration launched in March 2002 [Tapley et al., 2004]. The GRACE mission is designed around a microwave $\mathrm{K} / \mathrm{Ka}$ band ranging (KBR) system that observes small variations in distance between both satellites. The KBR data in combination with accelerometer and spaceborne GPS data from GRACE A and B are used to estimate independent monthly solutions of the Earth's gravity field. Tapley et al. [2004] demonstrated that GRACE is able to observe the gravitational effect caused by continental hydrology and that the spatial resolution of GRACE is sufficient to observe differences in water height between the Amazon and Orinoco river basins.

[3] Variations in the Earth's gravity field can be caused by a large number of processes within the Earth's interior or on its surface. The spatial and temporal resolution of a

\footnotetext{
${ }^{1}$ Faculty of Aerospace, Delft University of Technology, Delft, Netherlands.

${ }^{2}$ School of Civil Engineering, University of Newcastle, Newcastle, UK.

Copyright 2007 by the American Geophysical Union. 0148-0227/07/2006JB004882\$09.00
}

surface mass signal that can be observed by GRACE is limited for several reasons: (1) The gravity field is derived from observations that come with their own specific noise characteristics, (2) observations are collected at about $500 \mathrm{~km}$ above the surface, (3) sufficient time is required to cover the entire globe, and (4) KBR data are only acquired along the satellite flight path. For this study we used release 04 (RL04) of the monthly gravity field product distributed by the Center of Space Research (CSR) at the University of Texas at Austin which consists of 43 monthly coefficient sets starting in January 2003 and ending in September 2006.

[4] The monthly gravity solutions are affected by a north-south striping pattern which must be removed prior to interpretation of the GRACE data. For this we apply a postprocessing procedure recommended by Swenson and Wahr [2002] that is based on a isotropic and homogeneous Gaussian weighting function as described by Jekeli [1981]. This procedure is rather effective in suppressing the characteristic track pattern, yet the cause of the error is presently not well understood. Wahr et al. [2006] suggested two possibilities: category 1 errors are those that refer to GRACE observation noise and sampling, and category 2 errors depend on the quality of background correction models used to compensate short periodic mass variations. 
Table 1. Cumulative Variance Percentage ${ }^{a}$

\begin{tabular}{ccccccc}
\hline & \multicolumn{7}{c}{$\tau$} \\
\cline { 2 - 7 }$M$ & 4 & 5 & 6.25 & 7.5 & 8.75 & 10 \\
\hline 1 & 35.7 & 45.1 & 51.4 & 55.0 & 57.2 & 58.4 \\
2 & 47.2 & 57.3 & 64.0 & 67.7 & 69.9 & 71.3 \\
3 & 55.6 & 66.4 & 73.5 & 77.3 & 79.4 & 80.5 \\
4 & 61.3 & 70.6 & 76.7 & 80.2 & 82.1 & 83.2 \\
5 & 65.9 & 73.4 & 79.2 & 82.5 & 84.5 & 85.6 \\
6 & 68.7 & 76.0 & 81.4 & 84.5 & 86.3 & 87.5 \\
7 & 70.9 & 78.1 & 83.0 & 86.1 & 87.8 & 89.0 \\
\hline
\end{tabular}

${ }^{a}$ Explained by the EOF method as a function of smoothing radius $\tau$ and the EOF approximation level $M$ used to calculate the EQWHs.

The strategy currently followed by the CSR during the GRACE data processing is to remove short-period variations by means of an ocean tide model, an atmospheric pressure loading model and a barotropic ocean model [see also Flechtner, 2003].

[5] The main motivation for writing this paper is to identify signal and noise in the 43 monthly potential coefficients sets. We start in section 2.1 by showing that the first few empirical orthogonal functions (EOFs) capture a significant part of the surface mass signal. In section 2.2, properties of a synthetic error formed by the residuals after EOF compression are shown. Both signal and synthetic error are then compared to formal error estimates provided by the CSR in the form of coefficient standard deviations in their GGM02C model, and simulated background model errors for tides and atmospheric pressure variations. In section 3 we show that the computed surface mass signal results in crustal deformations that have a high correlation to vertical loading observed by a global network of GPS receivers. This technique provides a means to validate the computed GRACE surface mass signal map in a manner that is independent from observations used during the GRACE data processing. In section 4 it is mentioned that degree error spectra provide at best an impression of a global error that does not depend on geographic location or direction (so that we identify the error as homogeneous and isotropic). We focus on a refinement procedure which concerns the spatial structure of the predicted or formal GRACE errors that follow from a GRACE covariance matrix. This result is compared to correlation functions along parallels at $0^{\circ} \mathrm{N}$ which exhibit an alternating track correlation pattern in the GRACE data.

\section{Filter Method}

\subsection{Introduction}

[6] Gaussian smoothed equivalent water heights follow from

$$
H(\theta, \lambda)=\sum_{n m a} a_{e} \frac{(2 n+1)\left(\rho_{e} / \rho_{w}\right)}{3\left(1+k_{n}^{\prime}\right)} W_{n}(\tau) \bar{C}_{n m a}^{*} \bar{Y}_{n m a}(\theta, \lambda)
$$

where $W_{n}(\tau)$ contains the coefficients of Green function $W(\psi)$ that depends on the spherical distance $\psi \cdot W(\psi)$ is an approximation of a Gaussian convolution operator on the sphere as described by Jekeli [1981], who mentioned that $W(0)=1$ and $W(\tau)=1 / 2$, where $\tau$ is referred to as the smoothing radius. In equation (1), $\rho_{e}$ and $\rho_{w}$ are the average densities of the Earth and water, $a_{e}$ is the equatorial radius, $k_{n}^{\prime}$ are load Love numbers for an elastic lithosphere as explained by Farrell [1972], $\bar{C}_{n m a}^{*}$ are potential coefficients relative to a mean for which we used the average of all provided monthly coefficient sets, $\bar{Y}_{n m a}(\theta, \lambda)$ are normalized spherical harmonic functions of degree $n$, order $m$ and parity $a$ evaluated at colatitude $\theta$ and longitude $\lambda$.

[7] The next step is to store equivalent water heights (EQWH) grids as column vectors in a data matrix $D$ and to decompose this matrix into empirical orthogonal functions (EOFs). This data analysis method was first applied by Lorenz [1956], and it is easily implemented by means of a singular value decomposition (SVD) as described by Press et al. [1989]. After SVD we obtain

$$
D=U \Lambda V^{\prime}
$$

where the columns of $U$ and $V$ are orthonormal and where $\Lambda$ is a diagonal matrix with elements $\lambda_{i} \in \mathcal{R}$ and $\lambda_{i} \geq 0$. Three properties of EOFs are as follows: (1) columns vectors of the $U$ matrix are the eigenvectors of the "space" covariance matrix $D D^{\prime}$ while columns of $V$ are eigenvectors of the "time" covariance matrix $D^{\prime} D,(2)$ singular values stored in $\Lambda$ are the square roots of the eigenvalues of either the space and time covariance matrix whose geometric multiplicity is identical, (3) column vector $i$ in $U$ is said to describe a spatial mode that corresponds to singular value $\lambda_{i}$ while row vector $i$ in $V^{\prime}$ describes the evolution in time of mode $i$, and (4) in our case, there are 43 modes and the percentage of variance of a particular mode is computed as $\lambda_{i}^{2} / T R\left(\Lambda^{2}\right) \times 100 \%$.

[8] In this paper we say that $D$ is approximated at level $M$ by a linear combination of EOFs 1 to $M$ for which we select the largest $\lambda_{i}$. This is equivalent to replacing $\Lambda$ by $\Lambda^{\#}$ whereby the first $M$ singular values are retained while the rest of the singular values is set to zero. In the sequel an approximation of $D$ is referred to as $D^{\#}$, and it is derived from $U \Lambda^{\#} V^{\prime}$.

[9] The cumulative variance percentages of the surface mass signal smoothed at radius $\tau$ and approximated at EOF level $M$ are shown in Table 1. Table 1 suggests that $73.5 \%$ of the variance is contained in EOF modes 1 to 3 when $\tau=6.25^{\circ}$. We exclude the $\bar{C}_{200}$ term in equation (1) because it generates an ellipsoidal pattern whose time evolution is hard to interpret; instead, we follow the recommendation by $\mathrm{M}$. Cheng et al. (personal communication, 2006), who suggests replacing $\bar{C}_{200}$ by a new value based on LAGEOS data analysis [see also Cheng and Tapley, 2004].

[10] Our initial assumption is that $D$ may be replaced by $D^{\#}$ whereby the EQWH signal is approximated at level $M=3$ using a smoothing radius $\tau=6.25^{\circ}$. This setting captures most of the known effects in the surface mass signal; seasonal variations in continental hydrology and surface mass change are clearly represented in Figures 1 and 2 with a total variance of $73.5 \%$. Figure 1 (top) shows the springautumn cycle which is mostly visible in the continental hydrology, Figure 1 (middle) shows the winter-summer cycle, and Figure 1 (bottom) shows the secular rate of change. Our first three EOFs confirm the results published by Tapley et al. [2004], Velicogna and Wahr [2005, 2006], Hinderer et al. [2006], and others. 

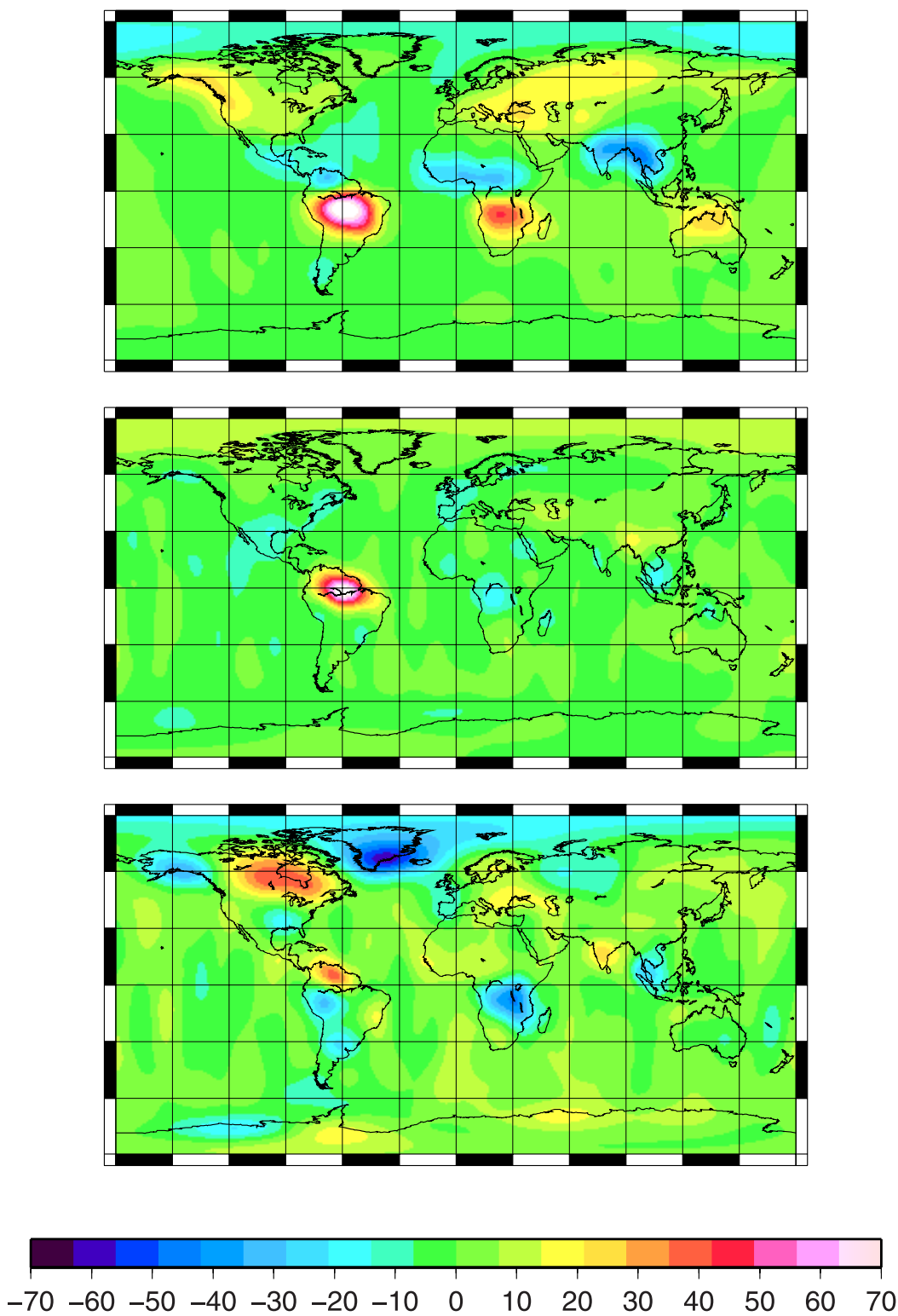

Figure 1. (top) EOF 1 displaying the spring-autumn cycle, (middle) EOF 3 indicating a winter-summer cycle, (bottom) EOF 2 representing the secular rate of change. The contribution of EOFs 1, 3, and 2 to the total variance is $51.4 \%, 9.5 \%$, and $12.6 \%$, respectively; for units, see Figure 2.

[11] The residual EOF signal described by modes 4 and onward is significantly affected by noise although we cannot exclude the possibility that this part contains geophysical signals. This issue is worked out in section 4 where we discuss the recovery of S2 tide aliasing errors and an semiannual hydrology signal. The sum of the residual EOF modes is called the synthetic EQWH error, and to verify the characteristics of this error, we conduct in section 2.1 additional tests to identify category 1 and 2 errors.

\subsection{Degree Spectra}

[12] In this section we discuss the spectral properties of the EQWH signal, synthetic EQWH errors that were introduced in section 2.1, formal errors based on an official GRACE gravity product (GGM02C) and simulated background model errors. We assume that all errors will be represented as degree error spectra and that all EQWH spectra are smoothed at the same smoothing radius $\tau$.

[13] The signal degree spectra are derived from $D^{\#}$ as discussed in section 2.1 while synthetic EOF error degree spectra are derived from $D-D^{\#}$. For this we take spherical harmonic decompositions of each column $k$ in $D^{\#}$ or $D-D^{\#}$ resulting in $H_{n m a, k}^{s}$ and $H_{n m a, k}^{r}$, respectively, where index $k$ relates to epoch $t_{k}$. In the next step the variances of $H_{n m a}^{s}$ and $H_{n m a}^{r}$ (hereinafter referred to as $\sigma^{2}\left(H_{n m a}^{s}\right)$ and $\left.\sigma^{2}\left(H_{n m a}^{r}\right)\right)$ are derived from $H_{n m a, k}^{s}$ and $H_{n m a, k}^{r} \forall k \in[1,43]$. The standard deviation by degree for the EQWH signal becomes

$$
\sigma_{s(n)}=\left(\sum_{m=n}^{N} \sum_{a=0}^{1} \sigma^{2}\left(H_{n m a}^{s}\right)\right)^{1 / 2}
$$



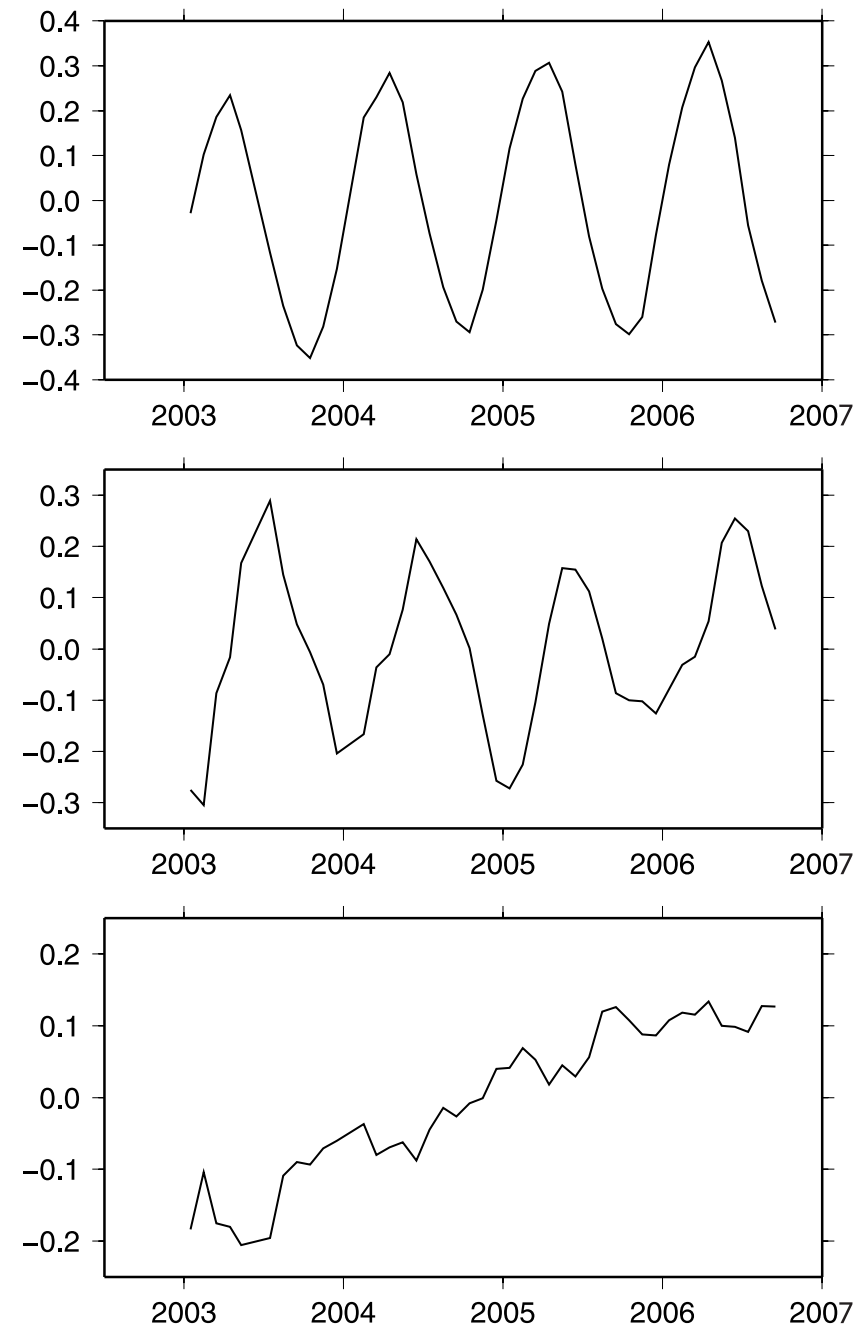

Figure 2. Time series belonging to the spatial EOF modes shown in Figure 1. To reconstruct each mode, these values should be multiplied by those in Figure 1 to yield centimeter surface water height thickness for Figures 2 (top) and (middle) and $\mathrm{cm} / \mathrm{yr}$ change signal for Figure 2 (bottom).

where $N$ is the maximum degree of the spherical harmonic expansion and $a$ is parity index relevant for spherical harmonics. In a similar way the standard deviation by degree for synthetic EQWH errors follows from $D-D^{\#}$ so that the standard deviation by degree for the synthetic EQWH errors becomes

$$
\sigma_{r(n)}=\left(\sum_{m_{n}}^{N} \sum_{a=0}^{1} \sigma^{2}\left(H_{n m a}^{r}\right)\right)^{1 / 2}
$$

[14] The signal and synthetic error derived from the EOF method will be compared to formal EQWH errors which are based on a two year mean GRACE combination gravity model (GGM02C). This model comes with diagonal variances for geopotential coefficients $\sigma^{2}\left(\bar{C}_{n m a}\right)$ so that the formal standard deviation becomes

$$
\sigma_{p(n)}=a_{e} \frac{(2 n+1)\left(\rho_{e} / \rho_{w}\right)}{3\left(1+k_{n}^{\prime}\right)} W_{n}(\tau)\left(\sum_{m=n}^{N} \sum_{a=0}^{1} \sigma^{2}\left(\bar{C}_{n m a}\right)\right)^{1 / 2}
$$

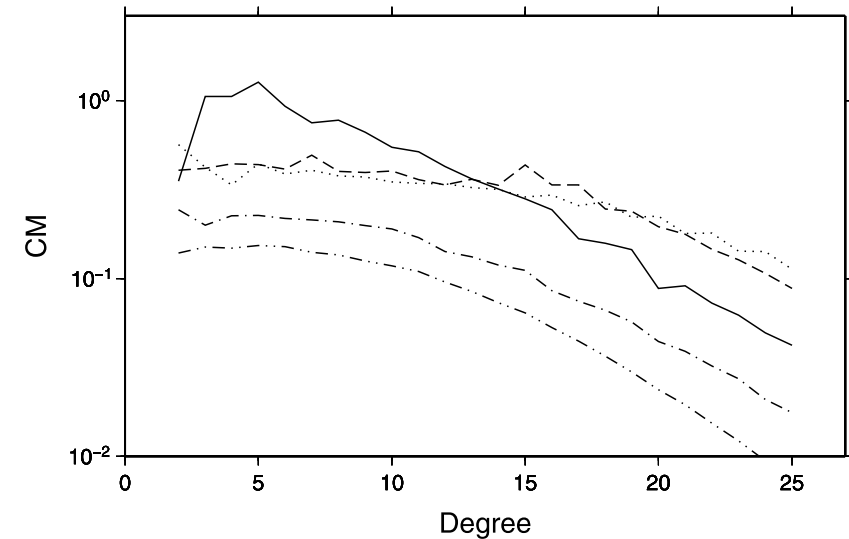

Figure 3. RMS in $\mathrm{cm}$ by degree for equivalent water heights smoothed at $6.25^{\circ}$ radius. The dashed line shows the noise in the GRACE time series, while the solid line describes the signal based on an EOF approximation using three modes. The dotted line is the formal RMS for EQWHs derived from diagonal variances provided with the GGM02C GRACE gravity model. A calibration factor of 0.85 was applied to the formal RMS by degree values. The dash-dotted line is an error estimate for tide errors based on the differences between FES2004 and GOT00.2, and the dash-dot-dotted line is an estimate for atmospheric pressure errors based on pressure differences derived from the ECMWF and the NMC.

[15] All three error curves are shown in Figure 3, where we also show the contribution of background model errors due to air pressure and ocean tide errors which will be explained in this section. In Figure 4 the standard deviations $\sigma_{s(n)}$ and $\sigma_{r(n)}$ and $\sigma_{p(n)}$ are represented as geoid heights without consideration of a smoothing radius.

[16] Figures 3 and 4 demonstrate that the synthetic EOF error is in agreement with formal errors up to degree 15 . An optimal agreement is obtained by a scaling factor of 0.85 applied to the formal standard deviation of the $\bar{C}_{n m a}$

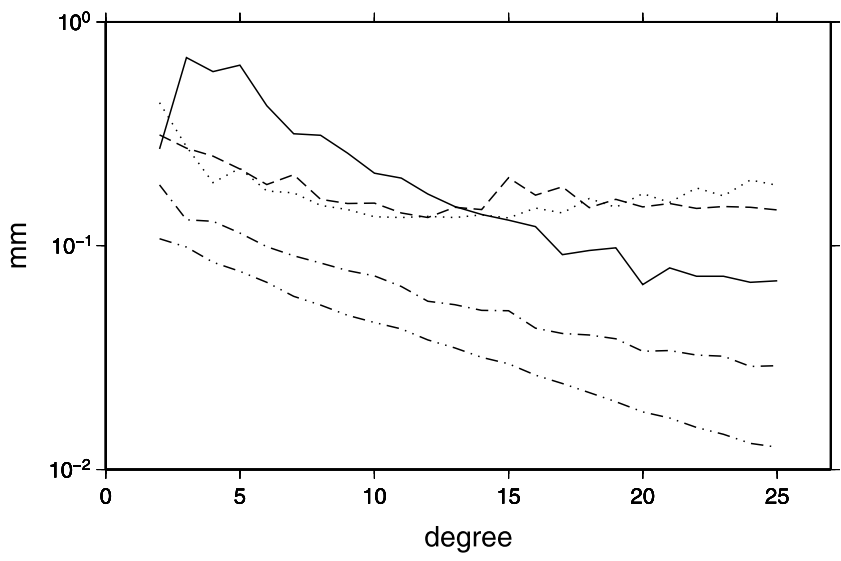

Figure 4. RMS in mm by degree for geoid heights similar to the cases shown in Figure 3. In this case we removed the smoothing effect; furthermore, a calibration factor of 0.85 was applied to the formal RMS values derived from the GGM02C model. 
coefficients for $n<25$. In this interval, Figure 3 shows that the EOF signal is well above formal errors, synthetic EOF errors and background model errors. Furthermore, we see a slightly elevated noise level between $n=15$ and 20 (and also at $n=30$ ) that is not predicted by GGM02C errors. Separate tests confirm that this effect is consistent with the $\mathrm{N}-\mathrm{S}$ banding pattern in the GRACE data and that potential coefficient errors increase for orders $m$ that approach $n$. The EOF method is capable of suppressing such features, although Figure 3 also suggests contamination of the retrieved signal around $n=15$.

[17] Up to now the signal and error discussion has been confined to an EOF analysis of the monthly gravity field product where we saw that synthetic errors and formal errors agree up to a scaling factor, see also Figures 3 and 4 . At best such an analysis shows that category 1 errors provided in the GRACE monthly gravity field product are in agreement with synthetic errors extracted from surface mass data. Category 2 errors, or background model errors (BMEs), are more difficult to quantify in the current GRACE product because a simulation of BMEs requires reprocessing of GRACE observation data, preferably twice with different background correction models that take away short periodic variations in the gravity field [see, e.g., Thompson et al., 2004; Schrama and Visser, 2006; Ray and Luthcke, 2006]. One of the conclusions of Schrama and Visser [2006] is that the largest BMEs can be expected from ocean tides and atmospheric pressure effects but that their errors are about an order smaller than the dominating continental hydrology signal in the GRACE data set. In this paper BMEs are assumed to be small and therefore BMEs are simplified in the sense that we obtain them directly as a difference of two competing background models that in turn are based on fundamentally different processing strategies. We compared the BME spectra for tides shown here to the approaches of both Schrama and Visser [2006] and Ray and Luthcke [2006] to conclude that there are no fundamental difference between these approaches for $n<25$.

[18] Figures 3 and 4 show an approximation of the tide BME which is computed as

$$
\sigma_{t(n)}^{2}=f_{n}^{2} \sum_{\omega, m a} \frac{1}{2}\left[\Delta P_{n m a, \omega}^{2}+\Delta Q_{n m a, \omega}^{2}\right]
$$

where $\omega$ is an index to summarize over eight dominant tidal constituents in the FES2004 tide model and where the choice of $f_{n}$ depends on the conversion of tidal heights into smoothed equivalent water heights (in which case $\left(f_{n}=\right.$ $\left.W_{n}(\tau)\right)$ or the conversion of tidal heights into geoid errors in which case $f_{n}$ follows from equation (1). In either case $\Delta P$ and $\Delta Q$ are in-phase and quadrature spherical harmonic coefficients differences between the FES2004 model which is an update from Lefèvre et al. [2002] and the GOT00.2 model which is an update of Schrama and Ray [1994]. We remark that both models are based on entirely different processing strategies although they are also based on identical altimeter data sets.

[19] Most of the differences between FES2004 and GOT00.2 appear in coastal zones, and above the T/P inclination latitude. We conclude from Figures 3 and 4 that tide BME are about a factor 3 smaller at degree 2-5 and a factor 10 smaller at degree 20-30 than residual errors derived from the GRACE data. We do get more significant tide BMEs if earlier versions like FES95.1 or prelaunch $\mathrm{T} / \mathrm{P}$ models are used although such scenarios are considered to be unrealistic.

[20] Figures 3 and 4 also include approximations of the surface level pressure BME. These curves are simulated by the mass effect of mean sea level pressure differences $\Delta P_{0}$ over land from the European Centre for Medium-Range Weather Forecasts (ECMWF) ERA40 reanalysis data and the National Centers for Environmental Prediction (NCEP) data between 1990 and 2000 excluding the years 1995 and 1996 (because of software transitions in the meteorologic products) and excluding air pressure difference poleward of $75^{\circ}$ latitude where both meteorologic models systematically differ as is explained by Schrama and Visser [2006]. The terrain mass effect relevant for our study is obtained from the barometric relation $\Delta P_{z}=\Delta P_{0} \exp \left(-z / z_{s}\right)$, where $z$ is the terrain height and $z_{s}$ the scale height of the standard atmosphere. From this simulation we conclude that the air pressure BME is a factor 3 smaller than the retrieved error estimates in the GRACE data. Furthermore, the mean sea level pressure BMEs decrease at a different rate in Figure 4 than the simulated tide BMEs.

\section{GPS Vertical Loading}

[21] GPS data collected by the International GNSS Service (IGS) between 2003 and 2007 that correspond to the used GRACE observation set offer a possibility to verify the GRACE deformation loading signal which depends on the choice of $\tau$ and the EOF compression level. To derive the vertical loading deformation from the GRACE solutions, we use relations that resemble equation (1) but that involve $h_{n}^{\prime}$ load Love numbers; for details, see, for instance, Küsche and Schrama [2005].

[22] We use the IGS combination solution vertical coordinate residuals computed by R. Ferland (available at $\mathrm{ftp}: / /$ macs.geod.nrcan.gc.ca/pub/requests/sinex/) that are provided at weekly intervals. These data are rather noisy at periods shorter than a month where GRACE is unable to predict a vertical loading signal so that it is justified that the GPS data are smoothed before a comparison. Our preprocessing procedure fits a function to the GPS data capable of modeling periods longer than 3 months; furthermore, it removes air pressure loading by means of a NCEP reanalysis product described in section 2.2. Our calculated air pressure loading predictions were compared to the values provided by the Special Bureau for Loading (maintained at http://www.sbl.statkart.no/products/operational), and no differences were encountered that would significantly affect our results.

[23] Our goal is to find an optimum EOF approximation level and smoothing radius. We prefer a fixed number of IGS stations in the comparison and for this reason the procedure consists of two steps:

[24] Step 1 starts with all IGS station data collected between 2003 and 2007 where we accept only those stations which show a (preprocessed) vertical displacement signal as described before with a RMS difference relative to the monthly GRACE values of less than $3.0 \mathrm{~mm}$ while the 
Table 2. Number of GPS Stations ${ }^{\mathrm{a}}$

\begin{tabular}{cccccccc}
\hline & \multicolumn{7}{c}{$\tau$} \\
\cline { 2 - 7 }$M$ & 4 & 5 & 6.25 & 7.5 & 8.75 & 10 \\
\hline 2 & 51 & 49 & 49 & 48 & 52 & 51 \\
3 & 57 & $\mathbf{5 9}$ & $\mathbf{5 9}$ & $\mathbf{5 9}$ & 55 & 55 \\
4 & 57 & $\mathbf{5 9}$ & $\mathbf{5 9}$ & 55 & 55 & 53 \\
5 & 56 & 57 & 54 & 51 & 49 & 50 \\
6 & 50 & 54 & 57 & 56 & 55 & 54 \\
7 & 53 & 56 & 54 & 53 & 51 & 52 \\
\hline
\end{tabular}

${ }^{\text {a }}$ Stations that satisfy the criterion that their vertical motion signal differs no more than $3 \mathrm{~mm}$ from the GRACE prediction and that the correlation between these signals is greater than 0.5 . Bold values indicate the maximum number of stations acquired in the comparison.

correlation is greater than 0.5 . The number of stations that pass this test are shown in Table 2, and the conclusion is that a maximum is reached for a EOF compression level of 3 or 4 and smoothing radii of $5^{\circ}, 6.25^{\circ}$, or $7.5^{\circ}$.

[25] Step 2 in the comparison between GPS and GRACE is an attempt to refine the optimum by calculating the mean RMS of the differences between the reference station set found in step 1 for various EOF approximation levels and smoothing radii. Step 2 results in a new optimum, i.e., EOF approximation level and smoothing radius, allowing a repetition of step 2 until the result converges. The final result is displayed in Table 3 which shows an optimal EOF compression level of 3 and an optimal smoothing radius of $\tau=5$ or $\tau=6.25^{\circ}$

\section{Spatial Representation of Signal and Error}

[26] A global map of the surface mass signal standard deviation is presented in Figure 5, while the corresponding error based upon a GRACE covariance matrix for July 2003
Table 3. RMS of the Difference Between GPS and GRACE Inferred Loading Deformations ${ }^{\mathrm{a}}$

\begin{tabular}{ccccccc}
\hline & \multicolumn{7}{c}{$\tau$} \\
\cline { 2 - 7 }$M$ & 4.00 & 5.00 & 6.25 & 7.50 & 8.75 & 10.00 \\
\hline 2 & 1.9925 & 2.0010 & 2.0033 & 2.0112 & 2.0248 & 2.0450 \\
3 & 1.9487 & $\mathbf{1 . 9 1 7 6}$ & $\mathbf{1 . 9 1 5 9}$ & 1.9268 & 1.9461 & 1.9723 \\
4 & 1.9691 & 1.9326 & 1.9266 & 1.9416 & 1.9639 & 1.9906 \\
5 & 1.9822 & 1.9503 & 1.9748 & 1.9546 & 1.9676 & 1.9944 \\
6 & 2.0319 & 1.9957 & 1.9505 & 1.9467 & 1.9476 & 1.9725 \\
7 & 2.0132 & 1.9838 & 1.9641 & 1.9608 & 1.9715 & 1.9770 \\
\hline
\end{tabular}

${ }^{\mathrm{a}} \mathrm{RMS}$ are in $\mathrm{mm}$. Deformations are observed at 59 IGS stations found in Table 2. Bold values indicate the lowest obtained RMS values.

[cf. Luthcke et al., 2006] is shown in Figure 6. The latter map is the result of a variance propagation technique whereby we calculate matrix vector products like $\bar{p}^{\prime} Q \bar{p}$ where $Q$ is the provided GRACE covariance matrix and where $\bar{p}$ are vectors with partial derivatives that relate potential coefficients to smoothed EQWH values, see also equation (1).

[27] Figure 5 suggests that the EQWH signal is located over the continents and that EOF compression is capable of removing most of the stripes in the maps. Figure 6 shows that the provided covariance matrix predicts a zonal error structure peaking at $10 \mathrm{~mm}$ near the equator and less than $3 \mathrm{~mm}$ poleward of $70^{\circ} \mathrm{N}$ and $70^{\circ} \mathrm{S}$. The formal error pattern is mostly determined by ground track coverage which was optimal in July 2003. A worst case covariance matrix for July 2004 indicates that the formal EQWH errors could increase by a factor 2 .

[28] Another measure for the spatial representation of the EQWH error is the standard deviation of the signal contained in EOFs 4 and onward for $6.25^{\circ}$ smoothing as
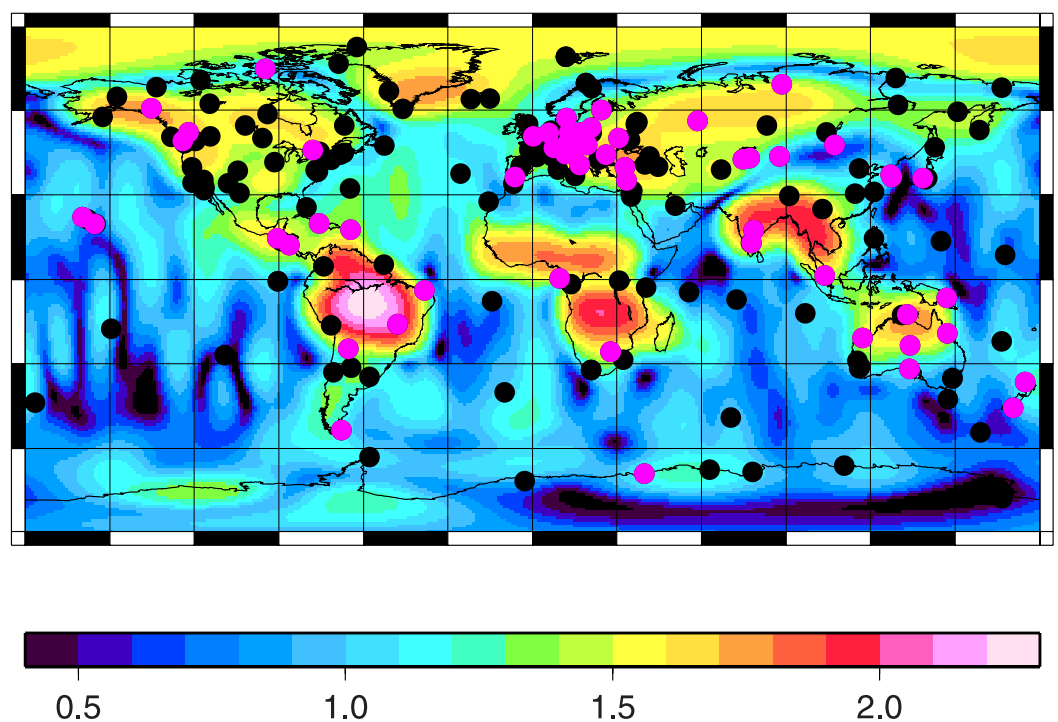

Figure 5. EQWH signal RMS as obtained by three EOF modes and a smoothing radius of $6.25^{\circ}$. The black circles represent all IGS stations, while the magenta circles indicate the reference station set that satisfies the condition that the RMS of the loading signal differences relative to GRACE values are less than $3 \mathrm{~mm}$ while the correlation is greater than 0.5 . The color scale is like $\log _{10}$ (signal) with "signal" in units of $\mathrm{mm}$. 

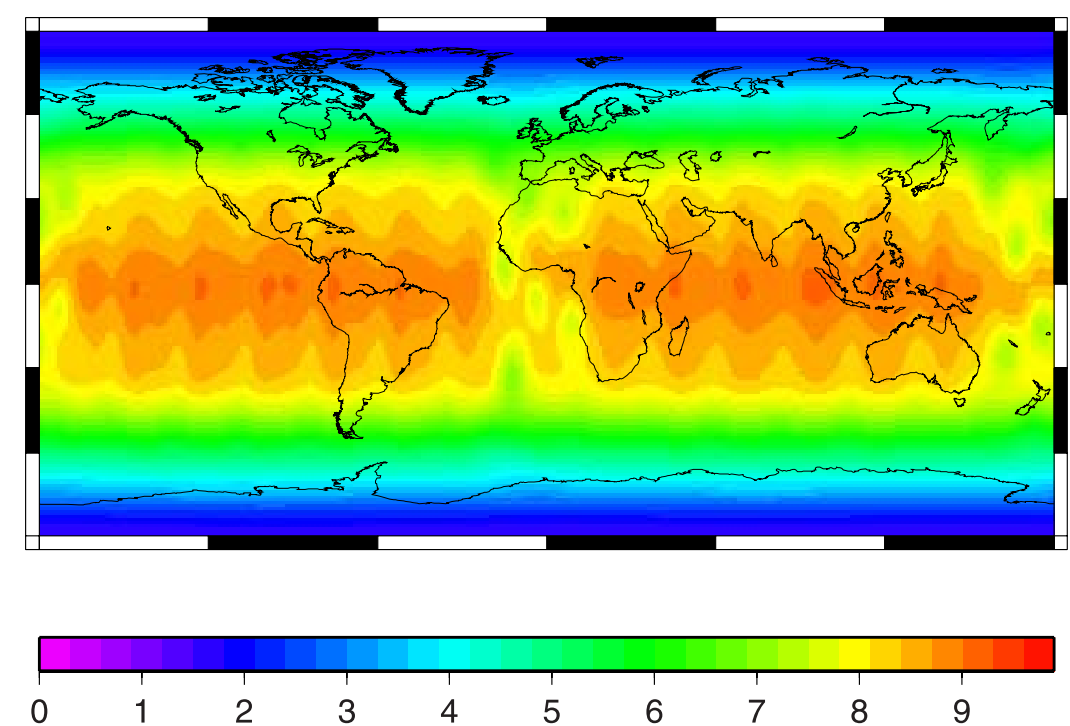

Figure 6. Predicted EQWH RMS as obtained from the July 2003 GRACE covariance matrix provided by F. G. Lemoine (personal communication, 2006). The propagated error is based on a smoothing radius of $7.5^{\circ}$; units are $\mathrm{mm}$.

is shown in Figure 7. In this case we do observe a number of remnant geophysical signals which do not show up in Figure 6 which will be discussed in section 4.1.

[29] By comparing Figures 6 and 7 in quiet regions which are less contaminated by geophysical signals we notice a different scaling factor. The geographical error predicted by the Goddard Space Flight Center (GSFC) July 2003 matrix peaks at $10 \mathrm{~mm}$ or so at the equator, and it peaks at 20 to $25 \mathrm{~mm}$ in Figure 7. In quiet polar regions the covariance matrix predicts less than $3 \mathrm{~mm}$ error where Figure 7 predicts less than $10 \mathrm{~mm}$. The conclusion is that there is a scale difference of about 2.5 to 3 meaning that the GSFC covariance matrix is too optimistic in representing geographic errors. F. G. Lemoine (personal communication, 2006) confirmed that their covariance matrix is not calibrated, the error described in the covariance matrix is therefore an internal representation of the EQWH error rather than that it is predicting residual signals as obtained by the EOF method.

\subsection{Remnant Geophysical Signals}

[30] In the following, we discuss the results of a pointwise frequency analysis of EQWHs contained in EOFs 4 and onward whereby we recognized the presence of 161 and 180 day signals. Separate tests have shown that the used CSR RL04 level 2 product is sufficiently long in time and sampled such that it is feasible to decouple the 161 day and the 180 day model parameters. The amplitudes of both features are shown in Figure 8.

[31] Ray and Luthcke [2006] explained that the S2 tide aliases to a 161 day cycle which is clearly visible in Figure 8 (top). The 161 day signal is well visible northwest of Australia, in the Weddell Sea, in the Argulhas retrore-

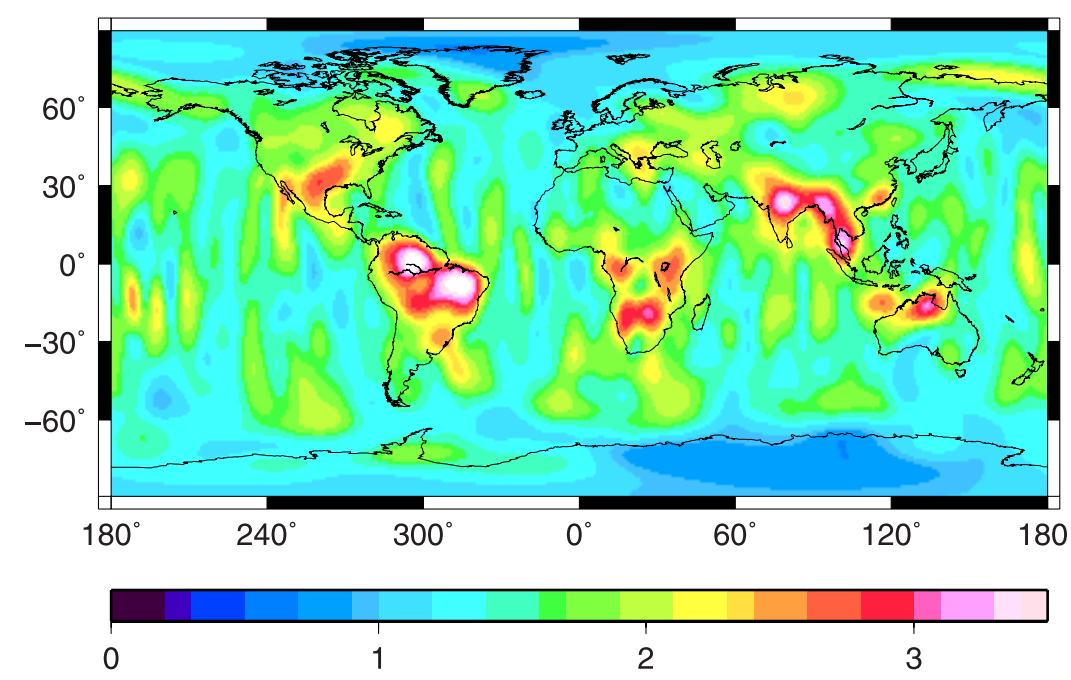

Figure 7. Surface water thickness RMS contained in EOF 4 and onward for $6.25^{\circ}$ smoothing. Units are $\mathrm{cm}$. 

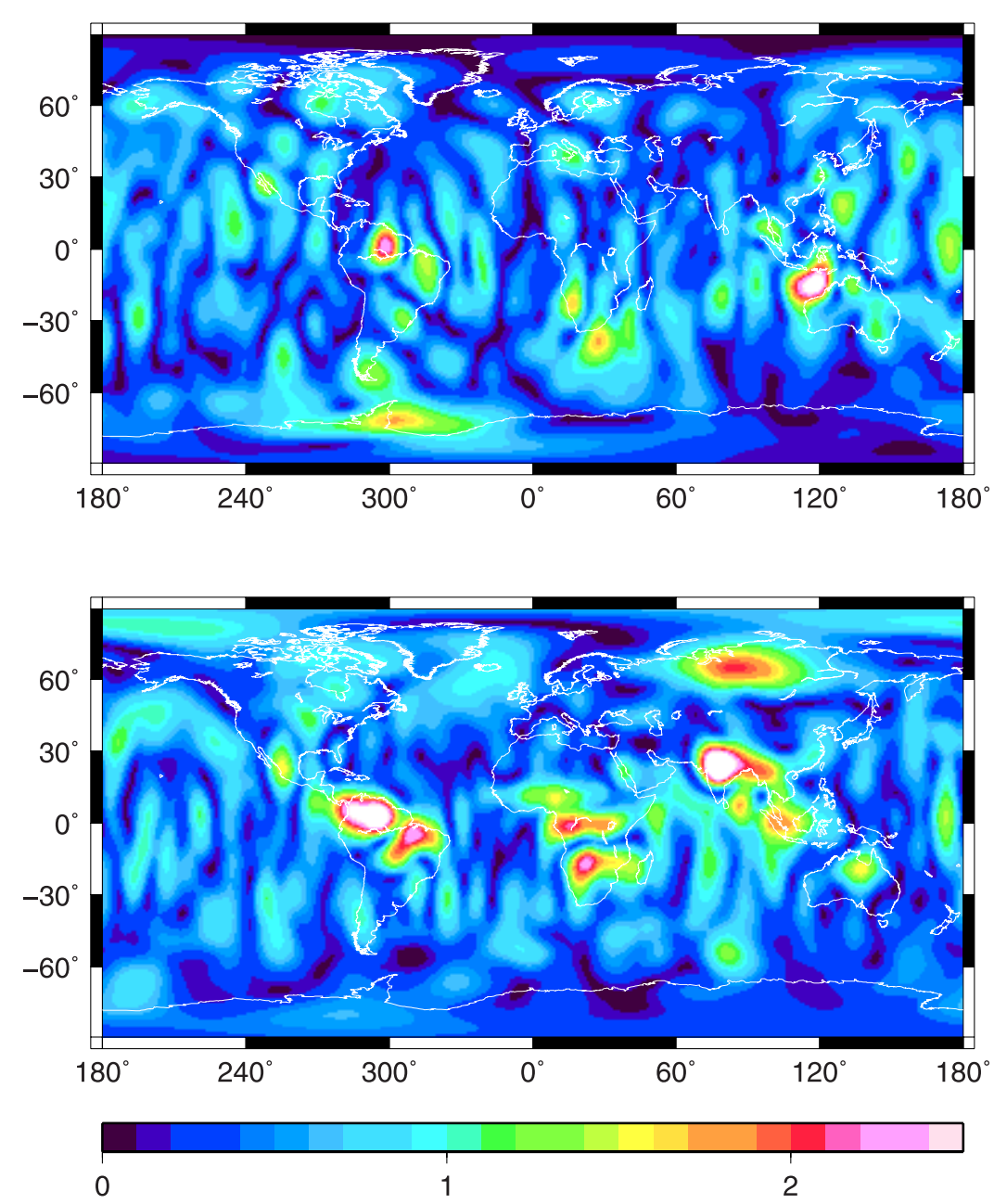

Figure 8. (top) Amplitude of the 161 day cycle which corresponds to the $\mathrm{S} 2$ tide aliasing into the GRACE data. (bottom) The 180 day cycle amplitude which is of hydrologic origin. Units are $\mathrm{cm}$.

flection area, and in the Patagonian shelf area. The reason for the S2 anomaly NW of Australia is not clear, and this could be an artifact in the FES2004 ocean tide model that was used as a background model in the GRACE data processing. Other 161 day features in Figure 8 are more easy to understand, the Weddell Sea is covered by perennial sea ice, and furthermore, it is at a latitude where it is not favorably mapped by satellite altimeter systems. The Patagonian region is one of the largest continental shelves and therefore it possesses complicated tidal dynamics, and the Argulhas region is known for its high mesoscale variability which may have affected the estimation of tidal constants from TOPEX/POSEIDON and Jason satellite altimetry data.

[32] The 180 day signal displayed in Figure 8 corresponds to the twice yearly continental hydrology signal and this signal is apparently not large enough to appear in one of the first three EOFs. The signal is of hydrologic origin because most of the energy in the GRACE observed EQWHs at a period of 180 days matches similar predictions shown in Figure 9 which follows from the Global Land Data Assimilation Systems (GLDAS) model described by Rodell et al. [2004].

[33] After removal of the 161 and the 180 day signals in the EOF residual signal we find mostly episodic events.
Most of these features do not have a clear geophysical origin, except for the Sumatra earthquake signal on 26 December 2004 [see also Han et al., 2006]. The Sumatra earthquake signal affects the estimation of a linear change signal and it is visible in our third EOF (see Figure 1). A second part of this signal (not shown here) appears in the residuals of EOF 4 and onward due to the episodic nature of this event.

\subsection{Alternating Track Errors}

[34] The final point to remark is that Figure 7 does reveal a typical north-south track pattern for instance in oceanic areas where we do not expect an EQWH signal larger than a few centimeters [see also Rietbroek et al., 2006]. Our experience is that the striping pattern results in signals that are more coherent along the tracks, while alternating track correlation patterns (ATCPs) appear between individual tracks.

[35] To demonstrate this feature, Figure 10 shows a longitudinal correlation function along a parallel centered at $0^{\circ} \mathrm{N}$ and a latitudinal correlation function centered at longitude $173^{\circ} \mathrm{E}$. Both functions show correlations between EQWH errors at the parallel or the meridian relative to $0^{\circ} \mathrm{N}$, $173^{\circ} \mathrm{E}$; the correlations are derived from the residual 


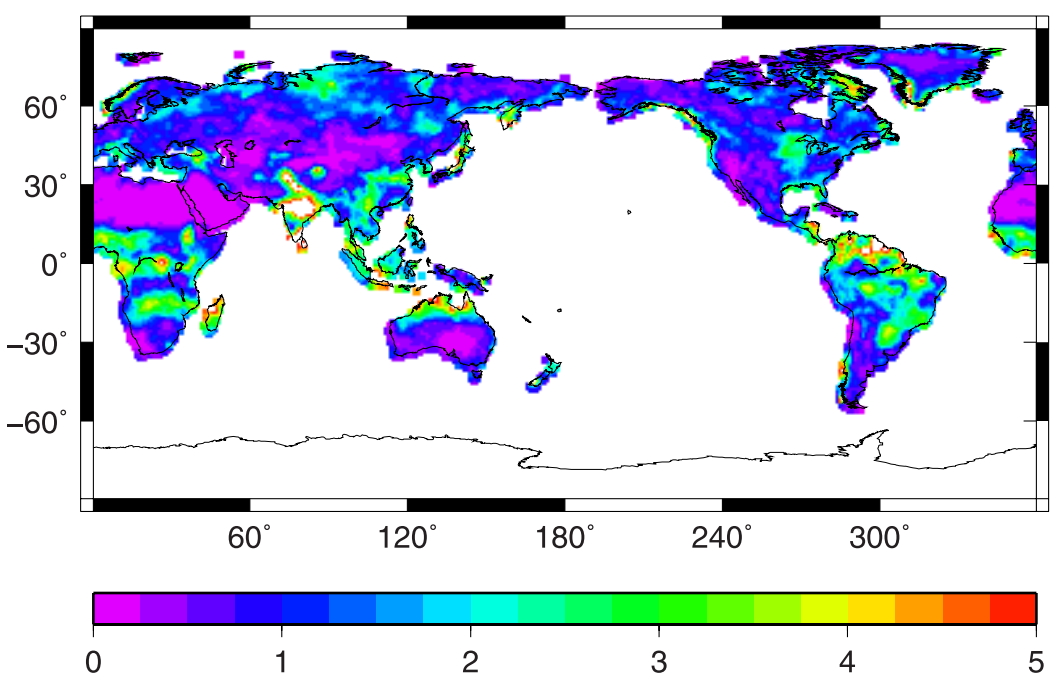

Figure 9. GLDAS hydrology amplitude at 180 day period, in $\mathrm{cm}$.

EQWHs smoothed at $6.25^{\circ}$ contained in EOF 4 and onward. Location $0^{\circ} \mathrm{N}, 173^{\circ} \mathrm{E}$ is chosen at one of the north-south going stripes in the equatorial Pacific which is far away from continental hydrology.

[36] The longitudinal correlation function along the $0^{\circ} \mathrm{N}$ parallel is represented by the solid line in Figure 10 (top), and it demonstrates that the GRACE level 2 CSR RL04 product is affected by an ATCP in the east-west direction whereas the correlations are smoother in the latitudinal direction. This ACTP behavior is best demonstrated near the equator and for latitudes up to $30^{\circ}$ where we count approximately 15 distinct bands in the horizontal correlation function resembling a degree and order 15 tesseral spherical harmonics pattern.

[37] Attempts to replicate the ATCP behavior by means of the GRACE covariance matrix for July 2003 [cf. Luthcke et al., 2006] are only partly successful; see also the dashed line in Figure 10 (top). The longitudinal correlation function derived from the July 2003 covariance matrix is able to represent correlations in the vicinity of $0^{\circ} \mathrm{N}, 173^{\circ} \mathrm{N}$ as is shown in Figure 10 , yet it is unable to predict a significant ATCP effect farther than about 3 times the smoothing radius $\tau$.

[38] The conclusion from this test is that the ATCP effect in the GRACE observed EQWHs may not be related to gravity model parameterization, i.e., if that were the case then ATCPs should have been predicted by the July 2003 covariance matrix. For this reason we suggest that future research focuses on other explanations for the ACTPs. Possible candidates are the presence of nuisance acceleration parameters in the precision orbit determination procedure including accelerometer scale and bias parameters. Both parameters play an important role in the processing of GRACE observation data, and any mismodeling of daily estimated nuisance parameters could cause a contamination effect that results in a tesseral banding pattern in the monthly gravity solutions.

\section{Conclusions}

[39] The purpose of this paper is to assess the signal and noise in the RL04 GRACE time variable gravity product released by the CSR which consists of 43 monthly potential coefficient sets starting in January 2003 and ending in September 2006. We separate signal and noise by means of an EOF approximation method to extract the most significant eigenvectors from the data covariance matrix
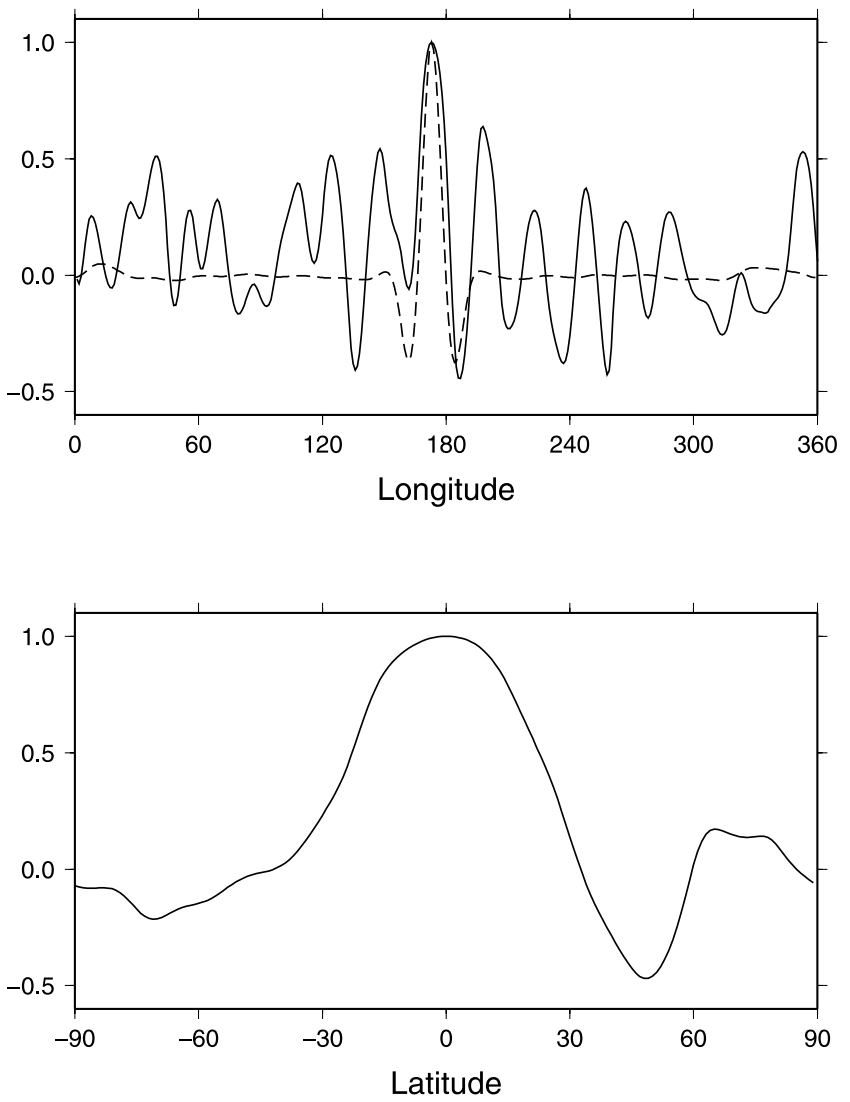

Figure 10. (top) An autocorrelation function (solid line) derived from the EQWH signal smoothed at $6.25^{\circ}$ contained in EOF 4 and onward along the $0^{\circ} \mathrm{N}$ parallel relative to $0^{\circ} \mathrm{N}$, $173^{\circ} \mathrm{E}$. The dashed line is predicted from the 3 July GSFC covariance matrix. (bottom) Corresponding meridional autocorrelation function. Units are dimensionless. 
that follows from the smoothed equivalent water height grids observed by GRACE. The success of the EOF approximation technique depends directly on an optimal choice of a smoothing radius and the number of EOF modes that represent the GRACE signal. Our conclusion is that a radius of $6.25^{\circ}$ and $3 \mathrm{EOF}$ modes are able to describe $73.5 \%$ of the variance.

[40] To validate signal and error estimates derived by EOF compression, we compute degree spectra which in turn are compared to the GGM02C model error estimates. A calibration scale factor of 0.85 is required to match the synthetic EOF errors and formal GGM02C model errors suggesting that the latter are too pessimistic.

[41] The present errors in the GRACE data are significantly larger than simulated background model errors derived from ocean tide and atmospheric pressure models. Degree error estimates derived from the EOF method reveal an elevated pattern in the GRACE data around degrees 15 and 30 which is consistent with a north-south banding pattern in the monthly gravity fields. The EOF filter method discussed in this paper is capable of removing striping effects although this discussion depends on the choice of an optimal smoothing radius and an EOF approximation level.

[42] An external validation of GRACE EQWHs obtained for $\tau=6.25^{\circ}$ and $3 \mathrm{EOF}$ modes relies on a comparison to the deformation loading signal observed by the IGS network. We found that 59 out of 202 IGS sites show a correlation greater than 0.5 and a RMS difference smaller than $3 \mathrm{~mm}$. The 59 selected GPS stations do show an optimal agreement with the GRACE data when we select smoothing radii at $5^{\circ}$ or $6.25^{\circ}$ using 3 EOFs.

[43] The residual signal contained in EOFs 4 and onward does contain remnant signals at periods of 161 and 180 days. The 161 day signal corresponds to the $\mathrm{S} 2$ tidal aliasing signal predicted by Ray and Luthcke [2006] which leads to S2 tide errors in the Weddell Sea, the Patagonian shelf and northwest of Australia. The 180 day signal is confirmed to match the 180 day periodicity in the GLDAS model described by Rodell et al. [2004]. Both signals are not energetic enough to stand out as individual EOFs and require a separate post processing after the EOF decomposition. The remainder of the residual EQWH signal freed from 161 and 180 day periodic signals does contain numerous episodic events including an Sumatra earthquake signal which also partially appears in our second EOF.

[44] Finally, we remark that the EQWH data contained in EOF 4 and onward do exhibit an alternating track correlation pattern (ATCP) that appears between individual northsouth going tracks. The ATCP is visualized by means of an autocorrelation function derived from the residual EOF signal in modes 4 and onward along a parallel at $0^{\circ} \mathrm{N}$ relative to $173^{\circ} \mathrm{E}$, where it resembles a tesseral harmonic feature corresponding to degree and order 15 . We are only partly successful in predicting the ATCP behavior by means of correlation functions derived from a GRACE covariance matrix. Our interpretation is therefore that the ATCP feature may be related to the handling of nuisance parameters in the GRACE data processing procedure at the CSR.

[45] Acknowledgments. The authors appreciate comments received from John Wahr and an anonymous referee that have helped to improve this manuscript, GRACE CSR RL04 data were obtained from the Center for
Space Research at the University of Texas at Austin, and meteorologic data were kindly provided by the European and American meteorologic organizations ECMWF and NMC. The generic mapping tools (GMT) developed by W. Smith and P. Wessel were used, and Frank Lemoine at the Goddard Space Flight Center in Greenbelt, Maryland, provided GRACE covariance matrices for July 2003 and 2004 .

\section{References}

Cheng, M., and B. D. Tapley (2004), Variations in the Earth's oblateness during the past 28 years, J. Geophys. Res., 109, B09402, doi:10.1029/ 2004JB003028.

Farrell, W. E. (1972), Deformation of the Earth by surface loads, Rev. Geophys., 10, 761-797.

Flechtner, F. (2003), AOD1B product description document, GRACE project documentation, JPL Rep. 327-750, Revision 1.0, Jet Propul. Lab. Pasadena, Calif

Han, S.-C., C. K. Shum, M. Bevis, C. Ji, and C.-Y. Kuo (2006), Crustal dilatation observed by GRACE after the 2004 Sumatra-Andaman earthquake, Science, 313, 658-662, doi:10.1126/science.1128661.

Hinderer, J., O. Andersen, F. Lemoine, D. Crossley, and J.-P. Boy (2006), Seasonal changes in the European gravity field from GRACE: A comparison with superconducting gravimeters and hydrology model predictions, J. Geodyn., 41, 59-68, doi:10.1016/j.jog.2005.08.037.

Jekeli, C. (1981), Alternative methods to smooth the Earth's gravity field, Rep. 327, Dep. of Geod. Sci. and Surv., Ohio State Univ., Columbus.

Küsche, J., and E. J. O. Schrama (2005), Surface mass redistribution inversion from global GPS deformation and Gravity Recovery and Climate Experiment (GRACE) gravity data, J. Geophys. Res., 110, B09409, doi:10.1029/2004JB003556.

Lefèvre, F., F. H. Lyard, C. Le Provost, and E. J. O. Schrama (2002), FES99: A global tide finite element solution assimilating tide gauge and altimetric information, J. Atmos. Oceanic Technol., 19(9), 13451356

Lorenz, E. N. (1956), Empirical orthogonal functions and statistical weather prediction, Inst. Arch. Noncirculat. Collect. 2, QC996.5.M41.M58 1, Dep. of Meteorol., Mass. Inst. of Technol., Cambridge.

Luthcke, S. B., D. D. Rowlands, F. G. Lemoine, S. M. Klosko, D. Chinn, and J. J. McCarthy (2006), Monthly spherical harmonic gravity field solutions determined from GRACE inter-satellite range-rate data alone, Geophys. Res. Lett., 33, L02402, doi:10.1029/2005GL024846.

Press, W. H., B. P. Flannery, S. A. Teukolsky, and W. T. Vetterling (1989), Numerical Recipes, the Art of Scientific Computing, FORTRAN Version, Cambridge Univ. Press, Cambridge, U.K

Ray, R. D., and S. B. Luthcke (2006), Tide model errors and GRACE gravimetry: Towards a more realistic assessment, Geophys. J. Int., 167(3), 1055-1059, doi:10.1111/j.1365-246X.2006.03229.X.

Rietbroek, R., P. LeGrand, B. Wouters, J.-M. Lemoine, G. Ramillien, and C. W. Hughes (2006), Comparison of in situ bottom pressure data with GRACE gravimetry in the Crozet-Kerguelen region, Geophys. Res. Lett., 33, L21601, doi:10.1029/2006GL027452.

Rodell, M., et al. (2004), The global land data assimilation system, Bull. Am. Meteorol. Soc., 85(3), 381-394.

Schrama, E. J. O., and R. D. Ray (1994), A preliminary tidal analysis of TOPEX/POSEIDON altimetry, J. Geophys. Res., 99, 24,799-24,808.

Schrama, E. J. O., and P. N. A. M. Visser (2006), Accuracy assessment of the monthly GRACE geoids based upon a simulation, J. Geod., 81, 6780, doi:10.1007/s00190-006-0085-1.

Swenson, S., and J. Wahr (2002), Methods for inferring regional surfacemass anomalies from Gravity Recovery and Climate Experiment (GRACE) measurements of time-variable gravity, J. Geophys. Res. 107(B9), 2193, doi:10.1029/2001JB000576.

Tapley, B. D., S. Bettadpur, J. C. Ries, P. F. Thompson, and M. M. Watkins (2004), GRACE measurements of mass variability in the Earth system, Science, 305, 503-505, doi:10.1126/science.1099192.

Thompson, P. F., S. V. Bettadpur, and B. D. Tapley (2004), Impact of short period, non-tidal, temporal mass variability on GRACE gravity estimates, Geophys. Res. Lett., 31, L06619, doi:10.1029/2003GL019285.

Velicogna, I., and J. Wahr (2005), Greenland mass balance from GRACE, Geophys. Res. Lett., 32, L18505, doi:10.1029/2005GL023955.

Velicogna, I., and J. Wahr (2006), Acceleration of Greenland ice mass loss in spring 2004, Nature, 443, 329-331, doi:10.1038/nature05168.

Wahr, J., S. Swenson, and I. Velicogna (2006), Accuracy of GRACE mass estimates, Geophys. Res. Lett., 33, L06401, doi:10.1029/2005GL025305.

D. Lavallée, School of Civil Engineering, University of Newcastle, Newcastle, NE1 7RU, UK. (d.a.lavallee@ncl.ac.uk)

E. J. O. Schrama and B. Wouters, Faculty of Aerospace, Delft University of Technology, Kluyverweg 1, NL-2629HS Delft, Netherlands. (e.j.o. schrama@tudelft.nl; bert.wouters@tudelft.nl) 